\title{
Development of a rapid and sensitive europium (III) chelate microparticle-based lateral flow test strip for the detection and epidemiological surveillance of porcine epidemic diarrhea virus
}

\author{
Jianbo Liu ${ }^{1}$ Hongyan Shi ${ }^{1}$ Guangyi Cong ${ }^{1}$. Jianfei Chen ${ }^{1} \cdot$ Xin Zhang $^{1} \cdot$ Da Shi $^{1} \cdot$ Liyan Cao $^{1} \cdot$ Xiaobo Wang $^{1}$.

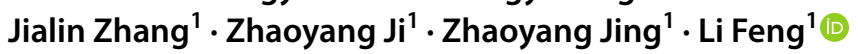

Received: 10 October 2019 / Accepted: 28 January 2020 / Published online: 6 March 2020

(c) Springer-Verlag GmbH Austria, part of Springer Nature 2020

\begin{abstract}
Porcine epidemic diarrhea virus (PEDV), a member of the genus Alphacoronavirus, is the predominant cause of severe enteropathogenic diarrhea in swine. A simple, rapid, specific, and sensitive method is critical for monitoring PEDV on pig farms. In this study, a simple and rapid lateral flow immunoassay detection system that integrates europium (Eu) (III) chelate microparticles was developed to identify PEDV in fecal swabs. This newly developed diagnostic sandwich immunoassay utilizes lateral flow test strips (LFTSs). The fluorescence peak heights of the test line (HT) and the control line (HC) were measured using a fluorescence strip reader, and the HT/HC ratio was used for quantitation. The limit of detection of PEDV with this LFTS was ??ten times the median tissue culture infectious dose $\left(\mathrm{TCID}_{50}\right)$ per $\mathrm{mL}$ ??. Fecal swab samples were used to determine the cutoff value. Field samples, various PEDV strains and other viruses were used to determine the sensitivity and specificity of the Eu (III) chelate microparticle-based LFTSs, which were $97.8 \%$ and $100 \%$, respectively, with a cutoff value of 0.05 , as compared with reverse transcription polymerase chain reaction (RT-PCR). In samples from piglets experimentally infected with PEDV, the results were in high agreement with those obtained by RT-PCR. Epidemiological surveillance of PEDV using the LFTSs ?? in areas threatened by African swine fever virus?? suggested that the PEDV positive rate on pig farms had significantly decreased, mainly due to the implementation of strict biosecurity measures. The results indicate that the Eu (III) chelate microparticle-based LFTS system is a rapid, sensitive, and reliable method for the identification of PEDV, indicating its suitability for epidemiological surveillance of PEDV infection.
\end{abstract}

\section{Introduction}

Enteric disease associated with porcine epidemic diarrhea virus (PEDV) was first reported in pigs in England in 1971 [20]. Since then, PEDV has spread to other European and Asian countries [23], as well as to North America in 2013 [8]. In China, PEDV was first confirmed in 1984, and since 2010, it has caused enteric disease, which is characterized by high morbidity and mortality in pre-weaning piglets, resulting in serious economic losses to the swine industry

Handling Editor: Diego G. Diel.

Li Feng

fengli@ caas.cn

1 State Key Laboratory of Veterinary Biotechnology, Harbin Veterinary Research Institute, The Chinese Academy of Agricultural Sciences, 678 Haping Road, Xiangfang District, Harbin 150069, China nationwide [5, 14]. After the initial introduction and outbreak of PEDV in the USA in April of 2013, sequencing of PEDV isolates (MN, IA1, IA2, USA/Iowa/16465/2013, and USA/Indiana/17846/2013) revealed a high degree of nucleotide sequence similarity ( $>99 \%$ identity) to a Chinese strain isolated from Anhui province (AH2012) whose genome sequence was submitted to the GenBank database in 2012 under the accession no. KC210145 [8, 24].

PEDV is an enveloped virus, belonging to the family Coronaviridae. It has a positive-stranded RNA genome that encodes four major structural proteins: the spike (S), nucleocapsid $(\mathrm{N})$, membrane, and envelope proteins. The PEDV N protein is important for maintaining the nucleocapsid structure and, other than the $\mathrm{M}$ protein of severe acute respiratory syndrome-related coronavirus, is the only coronavirus structural protein known to be phosphorylated $[1,25]$. The $\mathrm{N}$ protein is the predominant antigen expressed by coronavirus-infected cells [7,10], and detection of the $\mathrm{N}$ 
protein is therefore very helpful for the clinical diagnosis of PEDV infection.

Reverse transcription polymerase chain reaction (RTPCR) and real-time RT-PCR, as well as immunohistochemical techniques and enzyme-linked immunosorbent assay (ELISA) are the most common molecular techniques for the detection of PEDV antigens and antibodies [23]. Although these methods provide accurate results, they are not suitable for use by breeders on pig farms because of the need for well-trained technicians and specific equipment, in addition to the considerable amount of time and effort required. Recently, a lateral flow immunoassay, a well-established and accepted point-of-care testing technique, was used to develop quantitative fluorescent strips with various fluorescent reporters, which include colloidal gold, fluorescent dyes, quantum dots, and lanthanide chelates [2, 3, 27, 28]. The first case of African swine fever virus (ASFV) infection in China was reported on August 3, 2018 [26]. By July 10, 2019, almost all provinces in China had reported the appearance of ASFV (http://www.oie.int/). Since the initial outbreak of ASFV, strict biosecurity measures have been implemented to prevent further spread of the virus. In accordance with these measures, it is forbidden to transport swine tissues and serum as well as pork. Because of the limitations of techniques for the detection of PEDV, the aim of the current study was to develop a simple point-of-care technique to quantify the PEDV N protein in fecal swabs with the use of europium (Eu) (III) chelate microparticles and lateral flow test strips (LFTSs) for epidemiological surveillance of PEDV ??in areas threatened by ASFV??

\section{Materials and methods}

\section{Cells, viruses, reagents, and viral propagation}

Vero E6 cells (ATTC no. CRL-1586; American Type Culture Collection, Manassas, VA, USA) were maintained in Dulbecco's modified Eagle's medium (Gibco/Brl Division, Life Technologies, Inc., Grand Island, NY, USA) supplemented with $10 \%$ fetal bovine serum (Gibco/BRL) without antibiotic-antimycotic solution. PEDV strain LNCT2 (GenBank accession no. KT323980) was isolated and cultured in Vero E6 cells in our laboratory and then harvested as described by Oka et al. [19] and Liu et al. [17]. Trypsinethylenediaminetetraacetic acid (EDTA) was purchased from Gibco/BRL. All other chemicals were of reagent grade.

\section{Expression and purification of a recombinant PEDV $\mathrm{N}$ protein}

Two primers, PEDV-N-F1 (CTGGGATCCATGGCTTCT GTCAGTTTTCAG) and PEDV-N-R1326 (CCGCTCGAG
TTAATTTCCTGTGTCGAAG) were designed based on the sequence of the $\mathrm{N}$ gene of PEDV strain LNCT2. The restriction sites for endonucleases Bam HI and $\mathrm{XhoI}$ are underlined. The PCR reaction conditions were $98^{\circ} \mathrm{C}$ for $30 \mathrm{~min}$, followed by 30 cycles at $98{ }^{\circ} \mathrm{C}$ for $10 \mathrm{~s}, 60{ }^{\circ} \mathrm{C}$ for $30 \mathrm{~s}$, and $68{ }^{\circ} \mathrm{C}$ for $45 \mathrm{~s}$, and a final extension step at $68^{\circ} \mathrm{C}$ for $7 \mathrm{~min}$. The PCR products were $1326 \mathrm{bp}$ in length and purified using an E.Z.N.A. ${ }^{\circledR}$ Gel Extraction Kit (Omega Bio-Tek, Inc., Norcross, GA, USA) in accordance with the manufacturer's instructions. After digestion with the endonucleases BamHI and $\mathrm{XhoI}$, the products were cloned into the vector pGEX6p-1 (Invitrogen Corporation, Carlsbad, CA, USA), and the resulting construct was then used to transform competent Escherichia coli TOP10 cells (Tiangen Biotech (Beijing) Co., Ltd., Beijing, China). After sequencing, competent $E$. coli BL21 (DE3) cells were transformed with the recombinant plasmid pGEX-PEDV-N according to the product instructions. Expression of the $\mathrm{N}$ protein gene of PEDV from positive clones was confirmed by sodium dodecyl sulfate polyacrylamide gel electrophoresis (SDS-PAGE) and western blot analysis. The recombinant PEDV N protein was tagged with glutathione S-transferase (GST) for purification by affinity chromatography using nickel-nitrilotriacetic acid resin (QIAGEN, Hilden, Germany) according to the manufacturer's protocol.

\section{Preparation of monoclonal antibodies (mAbs) against the PEDV N protein}

Hybridoma cells secreting specific mAbs against the PEDV $\mathrm{N}$ protein were generated according to standard procedures, with some modifications [13]. Briefly, 6-week-old female $\mathrm{BALB} / \mathrm{c}$ mice were immunized subcutaneously with the purified recombinant PEDV N protein in Freund's complete adjuvant (Sigma-Aldrich Corporation, St. Louis, MO, USA). The mice received two booster immunizations with the $\mathrm{N}$ protein in Freund's incomplete adjuvant at 2-week intervals. One additional intraperitoneal immunization without adjuvant was administered. Three days after the final vaccination, sera were collected from the mice and used for ELISA and western blot experiments, as described in this report. Spleen cells were harvested and fused with SP2/0 myeloma cells at a ratio of 5:1 using polyethylene glycol 1450 (SigmaAldrich Corporation). The supernatants of surviving hybridoma clones were screened for reactivity and specificity using an indirect ELISA as described by Liu et al. [17], with minor modifications. The supernatants of the hybridoma clones $15 \mathrm{~B} 12$ and 4G7, which reacted strongly with the PEDV N protein in a western blot assay, were selected for further experiments. The protocols for all animal experiments in this study were approved by the Animal Care and Use Committee of our institute and performed in accordance with animal ethics guidelines. 


\section{Preparation of microparticles coupled with mAbs}

The conjugates of microparticles and anti-PEDV $\mathrm{N}$ mAbs (15B12) were prepared as follows: One $\mu \mathrm{g}$ of $210-\mathrm{nm} \mathrm{Eu}$ (III) chelate microparticles (Shanghai Femdetection BioTech Co., Ltd., Shanghai, China) was activated in $400 \mu \mathrm{L}$ of $0.05 \mathrm{M}$ borate buffer ( $\mathrm{pH} 8.0$ ) containing $30 \mu \mathrm{L}$ of a $10 \mathrm{mg} / \mathrm{mL}$ carbodiimide solution and $60 \mu \mathrm{L}$ of a $10 \mathrm{mg} / \mathrm{mL}$ $\mathrm{N}$-hydroxysuccinimide solution. After shaking for $15 \mathrm{~min}$ at room temperature, the mixture was centrifuged at $40,000 \times g$ for $10 \mathrm{~min}$ at $4{ }^{\circ} \mathrm{C}$. Then, the supernatant was discarded and the pellet was washed three times with $0.05 \mathrm{M}$ borate buffer (pH 8.0) and resuspended by sonication. Subsequently, $50 \mu \mathrm{g}$ of purified specific anti-PEDV-N mAb (15B12) was added. The labeling reaction was performed at room temperature for $2 \mathrm{~h}$. After the addition of $50 \mu \mathrm{L}$ of blocking buffer containing $250 \mu \mathrm{M}$ ethanolamine and $5 \mu \mathrm{g}$ of bovine serum albumin (BSA), the reaction was continued for $8 \mathrm{~h}$. Afterward, the mixture was centrifuged at $40,000 \times g$ for $10 \mathrm{~min}$. The supernatant was then discarded, and the pellet was washed three times with washing buffer, resuspended by sonication, and stored at $4{ }^{\circ} \mathrm{C}$ until analyzed.

\section{Preparation of test strips}

The Eu (III) chelate microparticle-based LFTSs were generated according to the procedures described by Liang et al. [16], with some modifications. Briefly, as shown in Fig. 1, the Eu (III) chelate microparticle-based LFTS is composed of five parts: a sample pad, a conjugate pad, a nitrocellulose (NC) membrane, an absorbent pad, and a backing plate. Glass fibers were cut into $25 \times 300$-mm pieces and immersed in phosphatebuffered saline ( $0.2 \mathrm{M}, \mathrm{pH} 8.0)$ containing $10 \mathrm{mM}$ EDTA, $1 \%$ Tween-20, $0.5 \%$ polyvinylpyrrolidone, and $0.5 \%$ bovine serum albumin (BSA) for $2 \mathrm{~h}$ at room temperature. Subsequently, the pieces were dried for $2 \mathrm{~h}$ at $37^{\circ} \mathrm{C}$ and stored in a dry package for use as the sample pad. The conjugate pad was made from glass fibers that were immersed in phosphate-buffered saline $(0.1 \mathrm{M}, \mathrm{pH} 7.4)$ containing $2.5 \%$ mycose, $1 \% \mathrm{BSA}, 1 \%$ Tween20 , and $0.5 \%$ polyvinylpyrrolidone for $2 \mathrm{~h}$ at room temperature

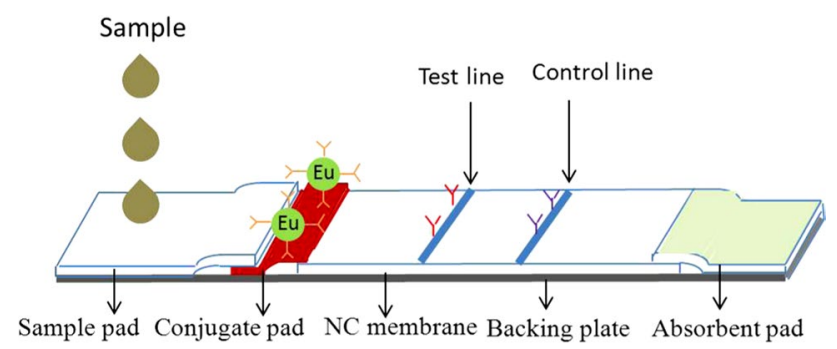

Sample Flow

Fig. 1 Schematic depiction of the construction of the LFTS and then dried for $2 \mathrm{~h}$ at $37^{\circ} \mathrm{C}$. The conjugate pad was covered with an appropriate volume of fluorescent microparticleconjugated anti-PEDV N mAbs (15B12) using an XYZ3060 dispense platform (BioDot, Inc., Irvine, CA, USA), dried at $37^{\circ} \mathrm{C}$ for $2 \mathrm{~h}$, and stored in a dry package. Another anti-PEDV $\mathrm{N} \mathrm{mAb}$ (4H7) was dispensed onto the $\mathrm{NC}$ membrane using the dispenser mentioned above to serve as the test line, while goat anti-mouse IgG polyclonal antibodies (pAbs) were dispensed on to the NC membrane, separated by a distance of $7 \mathrm{~mm}$, to serve as the control line. The mAb 4H7 and goat anti-mouse IgG pAbs were diluted with coating buffer to final concentrations of 0.025 and $1.0 \mathrm{mg} / \mathrm{mL}$, respectively. The NC membrane was dried at $37{ }^{\circ} \mathrm{C}$ for $2 \mathrm{~h}$ and stored in a dry package at room temperature for future use. The absorption pad was used without treatment. Then, the sample pad, conjugate pad, NC membrane, and absorbent pad were assembled onto the backing plate in the correct order, while ensuring that the ends of the four components overlapped to achieve a continuous flow from the sample pad to the absorbent pad. Subsequently, the whole plate was cut into 3-mm-wide strips, which were assembled in the strip cassettes.

\section{Test strip reader}

A fluorescence quantitative immunoassay reader (Shanghai Femdetection Bio-tech Co., Ltd.) was used to measure the heights of the fluorescence peaks of the test line (HT) and the control line (HC) of the Eu (III) chelate microparticlebased LFTSs. A fixed excitation light wavelength of $340 \mathrm{~nm}$ and an emission light wavelength of $620 \mathrm{~nm}$ were used to quantify the Eu (III)-chelated microparticles combined at the test line and control line.

\section{Fluorescence lateral flow assay procedure}

All rectal swab samples were diluted in $500 \mu \mathrm{L}$ of sample buffer (50 mM Tris [pH] 8.2, 1.0\% NaCl, 0.1\% Tween 20, $1.0 \%$ trehalose, and $1.0 \%$ sucrose). After mixing thoroughly, $100 \mu \mathrm{L}$ of the solution was dispensed onto the sample pad well of the lateral flow assay apparatus to detect the presence of the PEDV N protein, as described above. With this system, the liquid migrates towards the absorption pad by capillary action. After an 8-min reaction at room temperature, the test strip was inserted into the fluorescence quantitative immunoassay reader and the $\mathrm{HT}$ and $\mathrm{HC}$ values were determined.

\section{Determination of the cutoff values of the LFTSs using clinical samples}

A total of 108 rectal swab samples from piglets were collected from pig farms located in Heilongjiang province, China, and then subjected to RT-PCR and LFTS analysis. 
Total RNA was extracted from the samples using a Viral RNA Mini Kit (QIAGEN), and complementary DNA (cDNA) was synthesized using a PrimeScript ${ }^{\mathrm{TM}}$ II 1st Strand cDNA Synthesis Kit (Takara Bio, Inc., Otsu, Shiga, Japan) in accordance with the manufacturer's instructions. Two primers (PEDV-ORF3-F, 5'-GGAGCTCAATGTAGT TCCAA-3'; PEDV-ORF3-R, 5'-AGCTGCTTTACCATT GAGAA-3') were designed based on the sequence of open reading frame (ORF) 3 of PEDV strain LNCT2. The PCR reaction included an initial denaturation step at $94{ }^{\circ} \mathrm{C}$ for $2 \mathrm{~min}$, followed by 35 cycles of denaturation at $98^{\circ} \mathrm{C}$ for $10 \mathrm{~s}$, annealing at $58^{\circ} \mathrm{C}$ for $30 \mathrm{~s}$, and extension at $72{ }^{\circ} \mathrm{C}$ for $1 \mathrm{~min}$, with a final extension step at $72{ }^{\circ} \mathrm{C}$ for $7 \mathrm{~min}$. Afterward, the PCR products were separated on a $1.5 \%$ TAE (Tris base, acetic acid, and EDTA) agarose gel. Receiver operating characteristic (ROC) curves were constructed using MedCalc statistical software (version 9.2.1.0; https://www. medcalc.org/) to determine the cutoff value. Sample values below the cutoff value, as determined with the LFTS, were considered negative, and those above the cutoff value were considered positive.

\section{Evaluation of the specificity and sensitivity of the LFTS}

Different PEDV strains and other porcine viruses were used to evaluate the specificity of the LFTS. Briefly, PEDV strains CV777, a genotype 1 (G1) vaccine strain (GenBank accession no. KT323979), and LNCT2, a genotype 2 (G2) strain (GenBank accession no. KT323980; LNSY, accession no. KY007140; Hjms, accession no. KY007139), as well as other porcine viruses, including transmissible gastroenteritis virus (TGEV) strain H16 (GenBank accession no. FJ755618), porcine deltacoronavirus (PDCoV) strain $\mathrm{NH}$ (GenBank accession no. KU981062), and porcine rotavirus (PoRV) A (GenBank accession no. JF781161), were used to test the specificity of the LFTSs.

The sensitivity of the LFTSs was evaluated by comparing the limit of detection of PEDV strain LNCT2 with that of RT-PCR and an immunochromatographic assay (ICA) kit (BioNote, Inc., Hwaseong, Gyeonggi Province, South Korea). PEDV strain LNCT2 (?? $10^{5}$ times the median tissue culture infectious dose $\left[\mathrm{TCID}_{50}\right]$ per $\mathrm{mL}$ ??) was used as a positive control. Briefly, serial tenfold dilutions of PEDV strain LNCT2 were analyzed by RT-PCR, as well as with the LFTSs and ICA kit.

\section{Epidemiological surveillance of PEDV ??in areas threatened by ASFV??}

From January to June of 2019, 15 pig farms in Heilongiiang, Inner Mongolia, Shandong, Hebei, and Henan province were selected as locations for the surveillance of PEDV using the LFTSs and RT-PCR ??in areas threatened by ASFV??. Piglets with diarrhea were randomly selected from each pig farm, and PEDV surveillance was conducted using the LFTSs.

\section{Results}

\section{Identification of recombinant expression vectors}

The PEDV $\mathrm{N}$ gene was amplified from the genome of strain LNCT2. The PCR products were then inserted into the BamHI and XhoI cloning sites of the expression vector pGEX-6p-1. The desired recombinant plasmids were verified by DNA sequencing. The recombinant GST-PEDV-N protein was then successfully expressed in E. coli BL21 (DE3) cells, as verified by SDS-PAGE (Fig. 2).

\section{Production and characterization of $m A b s$ against PEDV}

Mice were immunized with the purified recombinant N protein to generate mAbs. Two hybridoma clones (15B12 and 4H7) secreted mAbs that reacted strongly with the PEDV $\mathrm{N}$ protein, as confirmed by western blot analysis (Fig. 3). Both the $15 \mathrm{~B} 12 \mathrm{mAb}$ and $4 \mathrm{H} 7 \mathrm{mAb}$ had the IgG1 heavy chain subtype, and the kappa light chain subtype, as determined using an SBA Clonotyping System-HRP kit (SouthernBiotech, Birmingham, AL, USA).

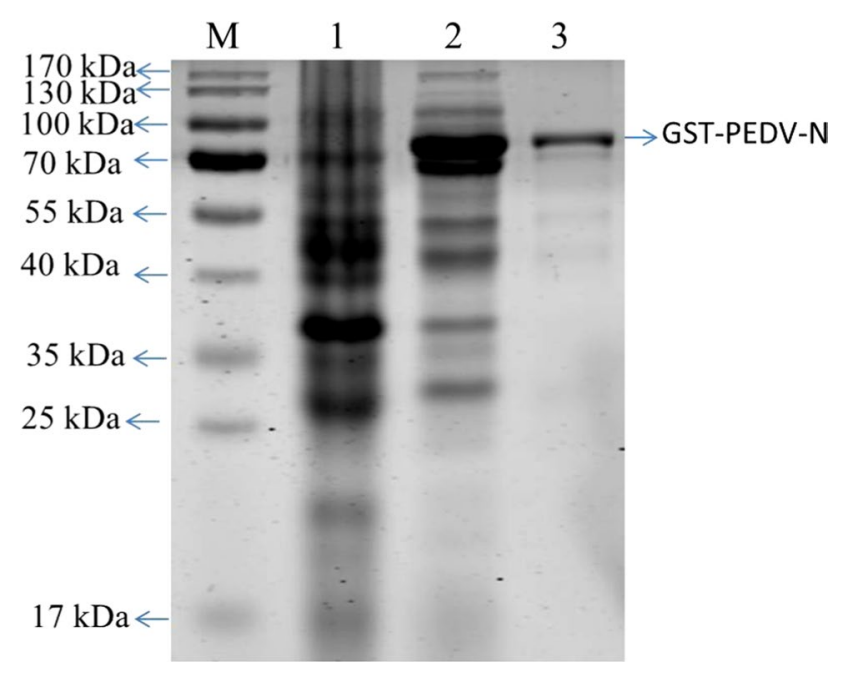

Fig. 2 Analysis of recombinant PEDV $\mathrm{N}$ protein expression by SDS-PAGE. Lane M, protein marker; lane 1, lysate of pGEXPEDV-N BL21 cells not induced by addition of isopropyl $\beta$-D-1thiogalactopyranoside (IPTG); lane 2, lysate of pGEX-PEDV-N BL21 cells induced by treatment with IPTG for $4 \mathrm{~h}(2)$; lane 3: purified of GST-PEDV-N. The native PEDV N protein is $\sim 51 \mathrm{kDa}$ in size, while the GST tag is $26 \mathrm{kDa}$ for a total of $77 \mathrm{kDa}$, for the tagged protein 


\section{The principle of the LFTS}

The LFTS was used to quantify PEDV by the method described by Liang et al. [16], with some modifications. Briefly, the LFTS using Eu (III) chelate microparticles was based on a conventional sandwich immunoassay. After the sample was added to the sample pad, the analyte migrated to the conjugate pad. The samples containing the PEDV $\mathrm{N}$ protein were combined with $\mathrm{mAb} 15 \mathrm{~B} 12$ labeled with $\mathrm{Eu}$ (III) chelate microparticles. After migrating to the test line, the complexes were captured by the anti-PEDV $\mathrm{N} \mathrm{mAb}$ $4 \mathrm{H} 7$, which was coated onto the $\mathrm{NC}$ membrane and formed $\mathrm{Eu}$ (III) chelate microparticle-labeled mAb-antigen-mAb sandwich complexes. Then, the labeled microparticles were immobilized at the test line, while the excess Eu (III) chelate microparticle-labeled mAb 15B12 continued moving along the membrane and was captured by the goat anti-mouse IgG pAbs at the control line. After the reaction, the LFTS was analyzed using a fluorescence quantitative immunoassay reader by measuring the fluorescence peak heights of the test and control lines (HT and $\mathrm{HC}$ values, respectively). Based on these results, the concentrations of the PEDV N protein of the samples were directly proportional to the HT/ $\mathrm{HC}$ ratio.

\section{Cutoff values for the LFTS}

The presence of the $\mathrm{N}$ protein in 108 rectal swab samples from piglets was detected using the LFTS and RT-PCR. Of these samples, 62 were negative by RT-PCR and 46 were positive (Table 1). The RT-PCR assay is considered the gold standard for establishing an ROC curve. The cutoff value was determined as shown in Fig. 4. The area under the ROC curve was 0.999 with a standard error of 0.003 . The cutoff value was 0.05 . Therefore, samples tested using the LFTS with values of $<0.05$ were judged to be negative, and those greater than the cutoff value were counted as positive.

\section{Specificity, sensitivity, and comparison assay}

As determined by the ROC curve, the sensitivity and specificity of the Eu (III) chelate microparticle-based LFTSs were $97.8 \%$ and $100 \%$, respectively (Fig. 4). The assay specificity was determined using PEDV and other viruses (i.e., TGEV, PDCoV, and PoRV). As shown by the LFTS values listed in Table 2, only the PEDV strains gave values above the cutoff value (0.05), indicating that the LFTS had good specificity at a cutoff value of 0.05 . Serial tenfold dilutions of PEDV strain LNCT2 (?? $\left.10^{5} \mathrm{TCID}_{50} / \mathrm{mL} ? \mathbf{?}\right)$ were analyzed by RT-PCR, as well as with the LFTS and ICA kit to determine the sensitivity of the LFTS. According to the results shown in Fig. 5, the LFTS had a detection limit of $10 \mathrm{TCID}_{50} / \mathrm{mL}$ of PEDV, while the detection limit of RT-PCR and the ICA kit was $100 \mathrm{TCID}_{50} / \mathrm{mL}$.

Table 1 The results for 108 samples detected by LFTS and RT-PCR that were used to determine the cutoff value of the LFTS using the ROC curve in MedCalc statistical software

\begin{tabular}{lccr}
\hline LFTS & \multicolumn{2}{l}{ RT-PCR } & Total \\
\cline { 2 - 3 } & Positive & Negative & \\
\hline Positive & 45 & 0 & 45 \\
Negative & 1 & 62 & 63 \\
Total & 46 & 62 & 108 \\
\hline
\end{tabular}

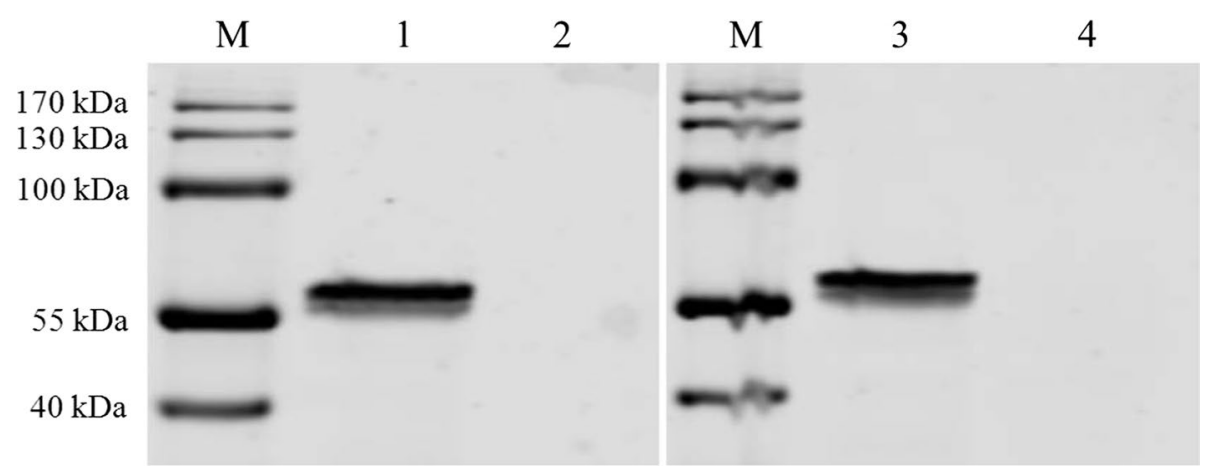

Fig. 3 Identification of mAbs (15B12 and 4H7) that are immunoreactive to the PEDV $\mathrm{N}$ protein expressed in Vero E6 cells in western blot analysis. Lane M, protein marker. The Vero E6 cells in lanes 1 and 3 were transfected with pcDNA3.1-PEDV-N (the plasmid was constructed by Shi et al. [22]), which expresses the PEDV N pro- tein, whereas the cells in lanes 2 and 4 were transfected with the pcDNA3.1 vector as a control. mAbs $15 \mathrm{~B} 12$ and $4 \mathrm{H} 7$ reacted well with the PEDV N protein expressed in Vero E6 cells. Neither of the mAbs reacted with the Vero E6 cells 


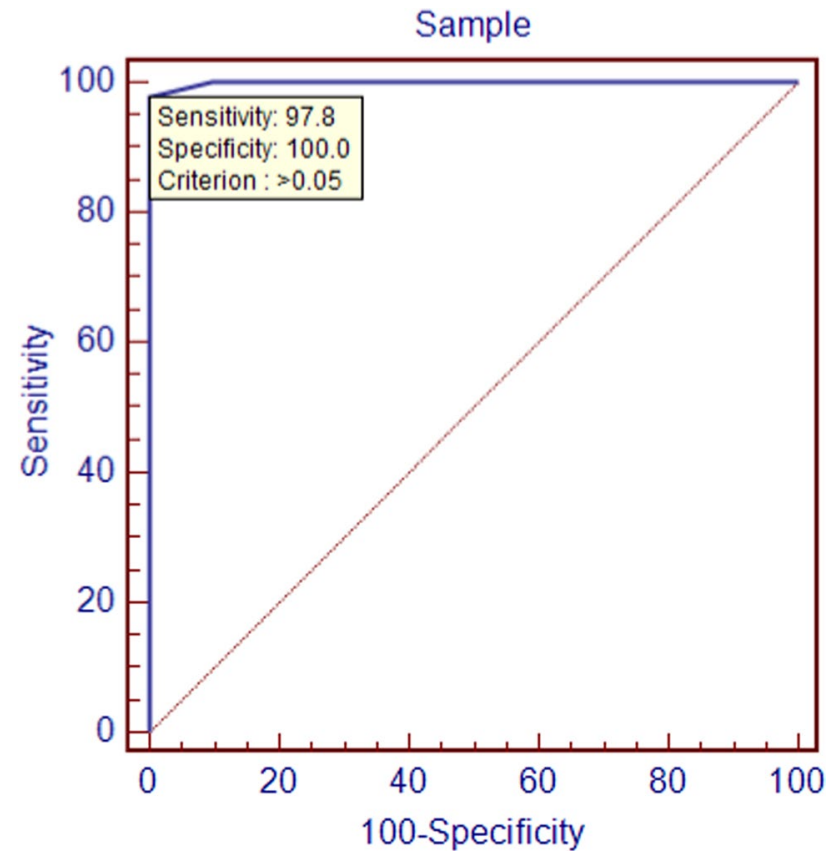

Fig. 4 Determination of the cutoff value and validation of the Eu (III) chelate microparticle-based LFTS by ROC. The Eu (III) chelate microparticle-based LFTS results for 108 confirmed rectal swab samples were compared with the RT-PCR results by ROC analysis. The cutoff value was 0.05 . The sensitivity of the Eu (III) chelate microparticle-based LFTS was $97.8 \%$, while the specificity was $100 \%$

\section{Epidemiological surveillance of PEDV ?? in areas threatened by ASFV??}

As shown in Table 3, a total of 923 piglets with diarrhea from 15 pig farms were selected to monitor PEDV by LFTS and RTPCR. The PEDV positivity rate of the LFTS was $7.0 \%$, while that of RT-PCR was $6.8 \%$. On seven pig farms, diarrhea was not caused by PEDV. The PEDV positivity rate on pig farms that experienced PEDV outbreaks was found to have declined significantly since the implementation of strict biosecurity measures to defend against the spread of ASFV.

\section{Discussion}

Since PEDV has become widespread in pig herds worldwide, many methods, such as RT-PCR [9], duplex RT-PCR [12], immunofluorescence analysis, immunohistochemical analysis, in situ hybridization [11], RT-loop-mediated isothermal amplification [21], and indirect ELISA, have been established to monitor viruses and antibodies $[4,6,15]$. However, the

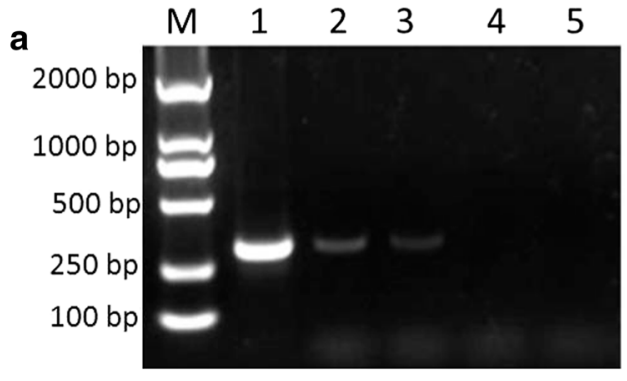

b
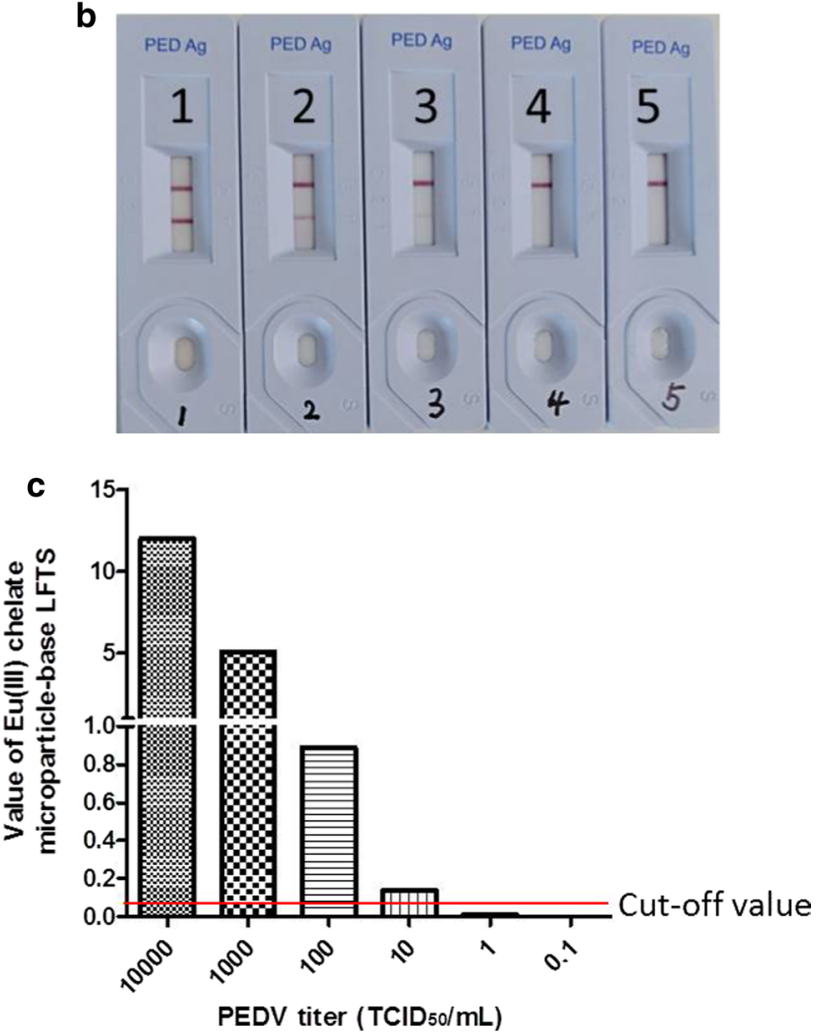

Fig. 5 Different PEDV dilutions detected by RT-PCR, the ICA strip, and the Eu (III) chelate microparticle-based LFTS. In (a), the ORF3 gene was amplified and the size was $283 \mathrm{bp}$. Lanes 1 to lane 5 in (a) and (b) are different dilutions $\left(10000,1000,100,10\right.$, and $1.0 \mathrm{TCID}_{50} /$ $\mathrm{mL}$, respectively). In (a) and (b), lanes 1 to 3 are positive for PEDV, while lanes 4 to 5 are negative. In (c), different PEDV sample dilutions were tested by the Eu (III) chelate microparticle-based LFTS. The red line indicates the suggested cutoff value. The figure in (c) was generated using GraphPad Prism 5 software (GraphPad Software, Inc., La Jolla, CA, USA)

above-mentioned methods are time-consuming and are mainly limited to use in the lab, with the exception of the ICA kit [18]. However, although the ICA kit is rapid, there is an important

Table 2 Results obtained with different viruses tested using the Eu (III) chelate microparticle-based LFTS

\begin{tabular}{llllllll}
\hline Virus & PEDV (CV777) & PEDV (LNCT) & PEDV (Hjms) & PEDV (LNSY) & TGEV & PoRV & PDCoV \\
\hline Value obtained using the LFTS & 13.41 & 12.25 & 12.71 & 21.94 & 0.00 & 0.00 & 0.00 \\
\hline
\end{tabular}


Table 3 Epidemiological surveillance of PEDV using LFTS and RT-PCR ??in areas threatened by ASFV??

\begin{tabular}{|c|c|c|c|c|c|c|}
\hline \multirow[t]{2}{*}{ Province } & \multirow[t]{2}{*}{ Pig farm no. } & \multirow{2}{*}{$\begin{array}{l}\text { No. of } \\
\text { diarrheic } \\
\text { piglets }\end{array}$} & \multicolumn{2}{|l|}{ LFTS } & \multicolumn{2}{|l|}{ RT-PCR } \\
\hline & & & $\begin{array}{l}\text { No. of PEDV-positive } \\
\text { piglets (positive rate) }\end{array}$ & $\begin{array}{l}\text { No. of PEDV-negative } \\
\text { piglets (??negative?? } \\
\text { rate) }\end{array}$ & $\begin{array}{l}\text { No. of PEDV-positive } \\
\text { piglets (positive rate) }\end{array}$ & $\begin{array}{l}\text { No. of PEDV-negative } \\
\text { piglets (negative rate) }\end{array}$ \\
\hline \multirow[t]{3}{*}{ Heilongjiang } & 1 & 78 & $0(0)$ & $78(100 \%)$ & $0(0)$ & $78(100 \%)$ \\
\hline & 2 & 85 & $0(0)$ & $85(100 \%)$ & $0(0)$ & $85(100 \%)$ \\
\hline & 3 & 63 & $14(22.2 \%)$ & $49(77.8 \%)$ & $13(20.6 \%)$ & $50(79.4 \%)$ \\
\hline \multirow[t]{3}{*}{ Shandong } & 4 & 43 & $9(20.9 \%)$ & $34(79.1 \%)$ & $9(20.9 \%)$ & $34(79.1 \%)$ \\
\hline & 5 & 56 & $3(5.4 \%)$ & $53(94.6 \%)$ & $3(5.4 \%)$ & $53(94.6 \%)$ \\
\hline & 6 & 71 & $10(14.1 \%)$ & $61(85.9 \%)$ & $10(14.1 \%)$ & $61(85.9 \%)$ \\
\hline \multirow[t]{3}{*}{ Inner Mongolia } & 7 & 68 & $7(10.3 \%)$ & $61(89.7 \%)$ & $7(10.3 \%)$ & $61(89.7 \%)$ \\
\hline & 8 & 56 & $11(19.6 \%)$ & $45(80.4 \%)$ & $10(17.9 \%)$ & $46(82.1 \%)$ \\
\hline & 9 & 77 & $0(0)$ & 77 (100\%) & $0(0)$ & $77(100 \%)$ \\
\hline \multirow[t]{3}{*}{ Hebei } & 10 & 95 & $0(0)$ & $95(100 \%)$ & $0(0)$ & $95(100 \%)$ \\
\hline & 11 & 42 & $6(14.3 \%)$ & $36(85.7 \%)$ & $6(14.3)$ & $36(85.7 \%)$ \\
\hline & 12 & 29 & $0(0)$ & $29(100 \%)$ & $0(0)$ & $29(100 \%)$ \\
\hline \multirow[t]{3}{*}{ Henan } & 13 & 75 & $0(0)$ & $75(100 \%)$ & $0(0)$ & $75(100 \%)$ \\
\hline & 14 & 36 & $5(13.9 \%)$ & $31(86.1 \%)$ & $5(13.9 \%)$ & $31(86.1 \%)$ \\
\hline & 15 & 49 & $0(0)$ & $49(100 \%)$ & $0(0)$ & $49(100 \%)$ \\
\hline Total & & 923 & $65(7.0 \%)$ & $858(93.0 \%)$ & $63(6.8 \%)$ & $860(93.2 \%)$ \\
\hline
\end{tabular}

imperfection. As the results of the ICA kit are judged by the appearance of a red color on the test line, it is sometimes difficult to judge whether the results are positive if the reaction is weak. Also, even when the samples contain large amounts of PEDV N protein, no quantitative estimates are possible, as the protein cannot be quantitated by color intensity. In the present study, an LFTS was established not only to screen for PEDV infection but also to quantify the results.

For the LFTS, Eu (III) chelate microparticles were used to label antibodies. The PEDV N protein in samples is captured by the Eu (III) chelate microparticle-labeled mAb 15B12 and forms $\mathrm{Eu}$ (III) chelate microparticle-labeled mAb-antigen complex, which can be captured by another mAb (i.e., 4H7) on the test line. The PEDV N protein is quantified by measuring the fluorescence peak heights of the test and control lines. The greater the HT/HC ratio, the more PEDV $\mathrm{N}$ protein is in the sample, providing an indication of the severity of the infection. As shown in Fig. 5, the detection limit of the LFTS was $10 \mathrm{TCID}_{50} / \mathrm{mL}$, with a better sensitivity than that of RT-PCR as well as the ICA kit (100 $\left.\mathrm{TCID}_{50} / \mathrm{mL}\right)$. Thus, the sensitivity of the Eu (III) chelate microparticle method is tenfold higher than that of the ICA kit. In this study, to improve performance, each microparticle contains thousands of Eu (III) chelates to amplify the signal.

PEDV has two genotypes, G1 and G2, with the main difference between the two are in the $\mathrm{S}$ gene. Although the $\mathrm{N}$ gene is conserved, two PEDV strains, CV777 and LNCT2 (G1 and $\mathrm{G} 2$, respectively), were used to test the LFTS. Both of these strains gave positive results, suggesting that the LFTS can be used to detect different PEDV strains. Since the limit of the LFTS was $10 \mathrm{TCID}_{50} / \mathrm{mL}$, the LFTS can be used to test for PEDV at a very early stage of infection, allowing measures such as strict biosecurity and targeted vaccination to be implemented to control the spread of PEDV.

The newly developed LFTS was used for epidemiological surveillance of PEDV. After the outbreak of ASFV in China, strict biosecurity measures were implemented nationwide, especially on pig farms. The sensitivity and specificity of traditional methods, such as PCR and RT-PCR, are rather limited. Rapid and sensitive methods such as colloidal gold and Eu (III) chelate microparticle fluorescence-based LFTS are easier to use for surveillance on pig farms. As shown in Table 3, the PEDV positivity rate on pig farms has significantly decreased due to the implementation of strict biosecurity measures and well as a decrease in breeding stocks.

In summary, a rapid, simple, and sensitive Eu (III) chelate microparticle fluorescence-based LFTS was developed to identify PEDV infection in pigs. The LFTS system has two main advantages: First, the cost of this simple and rapid assay is relatively low compared to conventional lateral flow strips. Second, the use of polystyrene Eu (III) chelate microparticles makes this assay more sensitive than RT-PCR. Thus, the polystyrene Eu (III) chelate microparticle-based LFTS is very suitable for the detection and monitoring of PEDV on pig farms. 
Acknowledgements This work was supported by grants from the National Natural Science Foundation of China (grant no. 31502066), Natural Science Foundation of Heilongjiang (grant no. QC2016043), 12th Five-Year National Key Technologies R\&D Program (grant no. 2015BAD12B02-6), and Innovation Capability Project for Research Institutions of Heilongjiang Province (grant no. YC2015D011).

\section{Compliance with ethical standards}

Conflict of interest None of the authors has a financial or personal relationship with other people or organizations that could inappropriately influence or bias the content of this report.

\section{References}

1. Brian DA, Baric RS (2005) Coronavirus genome structure and replication. Curr Top Microbiol Immunol 287:1-30

2. Cheng X, Pu X, Jun P et al (2014) Rapid and quantitative detection of C-reactive protein using quantum dots and immunochromatographic test strips. Int J Nanomedicine 9:5619-5626

3. Choi S, Choi EY, Kim DJ et al (2004) A rapid, simple measurement of human albumin in whole blood using a fluorescence immunoassay (I). Clin Chim Acta 339:147-156

4. Fan J, Zuo Y, Shen X et al (2015) Development of an enzymelinked immunosorbent assay for the monitoring and surveillance of antibodies to porcine epidemic diarrhea virus based on a recombinant membrane protein. J Virol Methods 225:90-94

5. Gao Y, Kou Q, Ge X et al (2013) Phylogenetic analysis of porcine epidemic diarrhea virus field strains prevailing recently in China. Arch Virol 158:711-715

6. Gimenez-Lirola LG, Zhang J, Carrillo-Avila JA et al (2017) Reactivity of porcine epidemic diarrhea virus structural proteins to antibodies against porcine enteric coronaviruses: diagnostic implications. J Clin Microbiol 55:1426-1436

7. Hou XL, Yu LY, Liu J (2007) Development and evaluation of enzyme-linked immunosorbent assay based on recombinant nucleocapsid protein for detection of porcine epidemic diarrhea (PEDV) antibodies. Vet Microbiol 123:86-92

8. Huang YW, Dickerman AW, Pineyro P et al (2013) Origin, evolution, and genotyping of emergent porcine epidemic diarrhea virus strains in the United States. MBio 4:1-8

9. Ishikawa K, Sekiguchi H, Ogino T et al (1997) Direct and rapid detection of porcine epidemic diarrhea virus by RT-PCR. J Virol Methods 69:191-195

10. Jayaram J, Youn S, Collission EW (2005) The virion N protein of infectious bronchitis virus is more phosphorylated than the $\mathrm{N}$ protein from infected cell lysates. Virology 339:127-135

11. Kim O, Chae C (2000) Comparison of reverse transcription polymerase chain reaction, immunohistochemistry, and in situ hybridization for the detection of porcine epidemic diarrhea virus in pigs. Can J Vet Res 66:112-116

12. Kim SY, Song DS, Park BK (2001) Differential detection of transmissible gastroenteritis virus and porcine epidemic diarrhea virus by duplex RT-PCR. J Vet Diagn Investig 13:516-520

13. Köhler G, Milstein C (1975) Continuous cultures of fused cells secreting antibody of predefined specificity. Nature 256:495-497
14. Li R, Qiao S, Yang Y et al (2014) Phylogenetic analysis of porcine epidemic diarrhea virus (PEDV) field strains in central China based on the ORF3 gene and the main neutralization epitopes. Arch Virol 159:1057-1065

15. Li Y, Zheng F, Fan B et al (2015) Development of an indirect ELISA based on a truncated $S$ protein of the porcine epidemic diarrhea virus. Can J Microbiol 61:811-817

16. Liang RL, Xu XP, Liu TC et al (2015) Rapid and sensitive lateral flow immunoassay method for determining alpha fetoprotein in serum using europium (III) chelate microparticles-based lateral flow test strips. Anal Chim Acta 891:277-283

17. Liu J, Shi H, Chen J et al (2017) Neutralization of genotype 2 porcine epidemic diarrhea virus strains by a novel monoclonal antibody. Virology 507:257-262

18. Lyoo KS, Yeom M, Kim J et al (2017) Development of rapid immunochromatographic strip test for the detection of porcine epidemic diarrhoea virus. Vet Rec 181(22):596-601

19. Oka T, Saif LJ, Marthaler D et al (2014) Cell culture isolation and sequence analysis of genetically diverse US porcine epidemic diarrhea virus strains including a novel strain with a large deletion in the spike gene. Vet Microbiol 173:258-269

20. Pensaert MB, de Bouck P (1978) A new coronavirus-like particles associated with diarrhea in swine. Arch Virol 58:243-247

21. Ren X, Li P (2011) Development of reverse transcription loopmediated isothermal amplification for rapid detection of porcine epidemic diarrhea virus. Virus Genes 42:229-235

22. Shi D, Lv M, Chen J et al (2014) Molecular characterizations of subcellular localization signals in the nucleocapsid protein of porcine epidemic diarrhea virus. Viruses 6(3):1253-1273

23. Song D, Park B (2012) Porcine epidemic diarrhoea virus: A comprehensive review of molecular epidemiology, diagnosis, and vaccines. Virus Genes 44:167-175

24. Stevenson GW, Hoang H, Schwartz KJ et al (2013) Emergence of porcine epidemic diarrhea virus in the United States: clinical signs, lesions, and viral genomic sequences. J Vet Diagn Investig 25:649-654

25. Zeng R, Ruan HQ, Jiang XS et al (2004) Proteomic analysis of SARS associated coronavirus using two-dimensional liquid chromatography mass spectrometry and one-dimensional sodium dodecyl sulfatepolyacrylamide gel electrophoresis followed by mass spectrometric analysis. J Proteome Res 3:549-555

26. Zhao D, Liu R, Zhang X et al (2019) Replication and virulence in pigs of the first African swine fever virus isolated in China. Emerg Microbes Infect 8(1):438-447

27. Zhang F, Zou M, Chen Y et al (2014) Lanthanide-labeled immunochromatographic strips for the rapid detection of Pantoea stewartii subsp. stewartii. Biosens Bioelectron 51:29-35

28. Zhu J, Zou N, Zhu D et al (2011) Simultaneous detection of highsensitivity cardiac troponin I and myoglobin by modified sandwich lateral flow immunoassay: proof of principle. Clin Chem 57:1732-1738

Publisher's Note Springer Nature remains neutral with regard to jurisdictional claims in published maps and institutional affiliations. 